\title{
Morphological properties and levels of extracellular peroxidase activity and light emission of the basidiomycete Armillaria borealis treated with $\beta$-glucosidase and chitinase
}

\author{
Mogilnaya OA*, Ronzhin NO, Artemenko KS and Bondar VS \\ Institute of Biophysics, Siberian Branch of Russian Academy of Sciences, Federal Research Center "Krasnoyarsk \\ Science Center SB RAS", 660036 Krasnoyarsk, Russia
}

Mogilnaya OA, Ronzhin NO, Artemenko KS, Bondar VS 2017 - Morphological properties and levels of extracellular peroxidase activity and light emission of the basidiomycete Armillaria borealis treated with $\beta$-glucosidase and chitinase. Mycosphere 8(4), 649-659, Doi 10.5943/mycosphere/8/4/11

\begin{abstract}
The study estimates morphological properties and levels of extracellular peroxidase activity and light emission of mycelium of the basidiomycete Armillaria borealis IBSO 2328 treated with $\beta$-glucosidase and chitinase. Mycelium incubated with the enzymes shows considerable morphological changes and indications of osmotic shock. Injuries observed in the cell envelope of the fungal hyphae are primarily attributed to the partial (in the $\beta$-glucosidase treatment) or complete (in the chitinase treatment) disintegration of the melanin layer on the surface of the cell wall. Changes in the cell wall of hyphae are accompanied by release of extracellular peroxidases of the fungus into the incubation medium and an increase in light emission relative to the luminescence of the control pellets. We assume that higher level of luminescence of the enzyme-treated mycelium samples could be related to the disintegration of the surface pigment layer of the hyphae and the partial loss of extracellular peroxidases. The data obtained confirm the previously proposed hypothesis in which light producing reaction of the fungus may be an additional way to neutralize active oxygen radicals under stress.
\end{abstract}

Key words - basidiomycetes - bioluminescence - cell wall - $\beta$-glucosidase - chitinase peroxidase

\section{Introduction}

Mycelium of basidiomycetes of the genus Armillaria is capable of emitting visible light (bioluminescence) with the maximum light emission at 520-530 nm (Shimomura 2006, Mihail \& Bruhn 2007, Medvedeva et al. 2014). Mycelium emits light when grown on the natural (wood) and artificial (nutrient medium) substrate. In Armillaria cultivated under laboratory conditions, bioluminescence is exhibited by film-like mycelium growing on solid and liquid nutrient media in Petri dishes and mycelium pellets growing in submerged culture under continuous orbital stirring (Mihail \& Bruhn 2007, Mihail 2013, 2015, Medvedeva et al. 2014, Mogilnaya et al. 2015). The studies referred to above showed that under stress conditions (incubation in water, mechanical irritation, mechanical injury), the intensity of light emission by mycelium increased. The stressor evidently affects the cell wall of the fungal hyphae. 
Each fungal hypha is surrounded by a cell wall and slime. The cell wall provides the hyphae with mechanical strength and protects them from changes of osmotic pressure and impacts of environmental stressors. At the same time, the cell wall is a dynamic structure, which constantly renews itself and changes in response to the surrounding conditions, enabling the hyphae to grow. The major components of the cell wall are chitin, $\beta$-D-glucan, and glycoproteins. Minor components include various proportions of lipids, proteins, and other elements. Chitin fibrils form a skeleton of considerable strength. The cell wall of the hyphae is covered by polysaccharide slime, which has pores and also consists of $\beta$-D-glucan (Bowman \& Free 2006, Feofilova 2010, Free 2013, Ene et al. 2015, Osinska-Jaroszuk et al. 2015, Fesel \& Zuccaro 2016). Branched glucans form a gel-like network in the hyphal envelope and intercellular space. This network retains the water necessary for the function of enzymes immobilized here (Ruel \& Joseleau 1991, Latge' \& Beauvais 2014).

Fungi of the genus Armillaria synthesize melanin at high quantity, but their amounts and distribution vary depending of the species. Pigments can be located within the hyphal cell wall, along the outer regions of the cell wall or on the outer cell wall surface. Melanin granules ranging from 40 to $100 \mathrm{~nm}$ in diameter are formed by sheets stacked in z-plane at distances of several angstrom. In the cell wall, melanin granules form layers too. The pigmented layer is not uniform, forming a mesh-like structure with pores ranging from 1 to $30 \mathrm{~nm}$ in diameter, through which small and large molecules can penetrate into cells. Melanin structures are bound to cell wall polysaccharides (Hegnauer et al. 1985, Zink \& Zink 1989, Eisenman et al. 2005, Zhong et al. 2008, Gessler et al. 2014, Nosanchuk et al. 2015). Melanin is believed to enhance the strength of the cell wall, have antioxidant properties, and be able to absorb active oxygen radicals, protecting hyphae from the effects of ultraviolet radiation (Free 2013). Koroleva et al. (2007) showed that basidiomycetes could synthesize humin-like substances. These substances, in contrast to melanin, which is firmly bound to the cell wall, are released into the environment.

It is well-known that abiotic (osmotic, temperature, hypoxic, $\mathrm{pH}$ ) stresses increase intracellular production of reactive oxygen species (ROS) (Tomanek 2015). To combat the damaging effects of ROS, basidiomycetes have a multicomponent antioxidant system, which includes, among other components, various peroxidases (Gessler et al. 2007, De Castro et al. 2013, Breitenbach et al. 2015). Numerous studies have identified secretory, extracellular, cytosolic, microsomal peroxidases, and ones localized in cell organelles. Most of these enzymes contain iron in the protoporphyrin ring of the active center. Because of the importance of heme-containing peroxidases to the life of basidiomycetes and the increasing interest in using them in bio- and nanotechnologies, much research effort has focused on these enzymes in recent years (Conesa et al, 2002, Wong 2009, Ruiz-Duenas \& Martinez 2010; Hofrichter et al. 2010, Knop et al. 2015, Carmona-Ribeiro et al. 2015, Pollegioni et al. 2015, Sáez-Jiménez et al. 2015).

In a previous study, we showed that pellets of $A$. borealis IBSO 2328 mycelium produced by growing the fungus in submerged culture emitted bright light and had rather low extracellular peroxidase activity (Mogilnaya et al. 2015). Results of that study suggested the following assumptions: (i) - pellets of mycelium of this fungus may contain low amounts of extracellular peroxidases and/or $\mathrm{H}_{2} \mathrm{O}_{2}$; (ii) - extracellular peroxidases of the fungus may be unavailable to exogenous substrates added to the reaction mixture for in vivo testing of peroxidase activity of mycelium.

The purpose of the present study was to investigate morphological properties and levels of extracellular peroxidase activity and light emission of Armillaria borealis IBSO 2328 mycelium treated with $\beta$-glucosidase and chitinase.

\section{Materials \& Methods}

\section{Material}

The luminous fungus Armillaria borealis IBSO 2328 is available in the CCIBSO 836 collection of the Institute of Biophysics, Siberian Branch of Russian Academy of Sciences. 


\section{Culture medium and procedures}

Mycelium pellets were produced in submerged culture of the fungus in PDB (HiMedia Laboratory, India) - potato extract $(200 \mathrm{~g} / \mathrm{L})$, dextrose $(20 \mathrm{~g} / \mathrm{L})$. The fungus was cultivated in 300 $\mathrm{ml}$ flasks containing $100 \mathrm{ml}$ nutrient medium, at a temperature of $24^{\circ} \mathrm{C}$ and continuous agitation at 160-180 rpm using an Environmental Shaker-Incubator ES-20 (BIOSAN, Latvia). Mycelium pellets were grown for 14 days. Suspension of crushed mycelium of A. borealis IBSO 2328 that had been grown in Petri dishes on PDA was used as inoculum.

At regular intervals, 2-3 flasks with pellets were taken to determine growth parameters of the culture. Glucose concentration in the culture medium during cultivation was measured by the glucose oxidase method, using an enzyme kit for measuring glucose ("Diakon DS", Russia). Before measurements, the culture medium was diluted 25-fold with deionized (DI) water. Deionized water was produced using a Milli-Q system (Millipore, U.S.).

\section{Luminescence measurement}

To measure luminescence, pellets were taken out of the nutrient medium and rinsed in DI water to remove residual nutrient solution and metabolites. Then, the level of light emission of the pellets was measured with a Glomax 20/20 luminometer (Promega, U.S.) calibrated using the Hastings - Weber radioactive standard (Hastings \& Weber 1963). One luminescence unit was 2.7 $\times 10^{3}$ photons per second. Specific luminescence activity of mycelium was determined as the ratio of its light emission to the dry weight of the pellets. To estimate the biomass, the pellets were dried in a rotational vacuum concentrator (Concentrator 5301, Eppendorf, Germany) at $60^{\circ} \mathrm{C}$ for several hours, until constant dry weight.

Additional experiments were performed to determine the time-course of changes in catalase and total peroxidase activities in A. borealis mycelium extracts during cultivation. Detailed methods of preparing pellet extracts and measuring activities of the enzymes can be found elsewhere (Mogilnaya et al. 2015).

\section{Enzymatic treatments of pellets and measurement of peroxidase activity}

At 7-9 days of cultivation, pellets were taken out of the nutrient medium and incubated with sweet almond $\beta$-glucosidase (EC 3.2.1.21) (Serva, Germany) or Streptomyces griseus chitinase (EC 33.2.1.14) (Sigma-Aldrich, U.S.). Enzyme solutions were prepared in $10 \mathrm{mM}$ PBS (pH 6.0). Pellets were placed in DI water that contained $\beta$-glucosidase $(0.5-1 \mathrm{IU} / \mathrm{ml})$ or chitinase $(0.74 \mathrm{IU} / \mathrm{ml})$ and incubated at $26^{\circ} \mathrm{C}$ for 4 hours. Control samples of mycelium were incubated in DI water under similar conditions but without enzymes. After incubation, we measured light emission intensities and levels of extracellular peroxidase activity of the control and treatment pellets. After removal of the control and treatment pellets, the incubation media were also analyzed for peroxidase activity.

Extracellular peroxidase activity of pellets in vivo and peroxidase activity on the incubation media were evaluated by using the azo coupling reaction (Mogilnaya et al. 2015, 2016). Peroxidase activity of native mycelial pellets was determined with some modifications: only phenol ( 0.56 $\mathrm{mg} / \mathrm{ml})$ and 4-aminoantipyrine (4-AAP) $(0.1 \mathrm{mg} / \mathrm{ml})$ were introduced into the reaction. In the experiment, peroxidase and $\mathrm{H}_{2} \mathrm{O}_{2}$ were not added to the reaction. After 1 hour of incubation of pellets at $25^{\circ} \mathrm{C}$, the dye solution was collected, and absorbance of chromogen was measured in a UV-1800 spectrophotometer (Shimadzu, Japan) at a wavelength of $506 \mathrm{~nm}$.

To determine peroxidase activity of the incubation medium, $1 \mathrm{mM} \mathrm{H}_{2} \mathrm{O}_{2}$ was added to it, along with phenol and 4-AAP.

\section{Visualization of luminescence and images of pellets}

Visual observation of luminescence of native pellets was conducted by using a ChemiDoc ${ }^{\mathrm{TM}}$ XRS System (Bio Rad, U.S.) in a dark cabinet, in the signal accumulation mode. Exposure time, 300 seconds, was chosen by trial and error. Images of pellets were made with a PowerShot S50 camera (Canon, Japan). 
Micrographs of the pellets and hyphae were obtained by using an AxioImager M2 (Zeiss, Germany) microscope in the transmitted and reflected light mode. Before microscopy, the pellets were fixed for 15 minutes in a $3.7 \%$ solution of paraformaldehyde prepared in $10 \mathrm{mM} \mathrm{PBS}(\mathrm{pH}$ 6.9), washed several times in buffer solution, and then rinsed in DI water. For fluorescent microscopy, the pellets were stained for 15 minutes in the aqueous solution of acridine orange dye $(20 \mu \mathrm{g} / \mathrm{ml})$.

Samples for electron microscopy were prepared as follows. Pellets of control and treatment samples were fixed in $2.5 \%$ glutaraldehyde in $0.1 \mathrm{M}$ cacodylate buffer ( $\mathrm{pH} 7.2)$, postfixed in $1 \%$ $\mathrm{OsO}_{4}$ in the same buffer, and dehydrated in graded ethanol and acetone solutions. The samples were embedded in epoxy resin mixture based on Epon 812 (Fluka, Germany). Ultrathin sections were cut using glass knives on Om U3 ultramicrotome (Reichert, Austria) and examined using an HT770 electron microscope (Hitachi, Japan) in the Krasnoyarsk Regional Joint Instrument Usage Center of SB RAS (KRJIUC FRC KSC RAS).

\section{Results}

\section{Morphology and luminescence}

Changes in the parameters investigated in this study (glucose concentration in the nutrient medium, fungal biomass increase, intensity of light emission by mycelium, and levels of catalase and total peroxidase activities in mycelium extracts) during submerged cultivation of $A$. borealis IBSO 2328 are shown in Fig. 1.
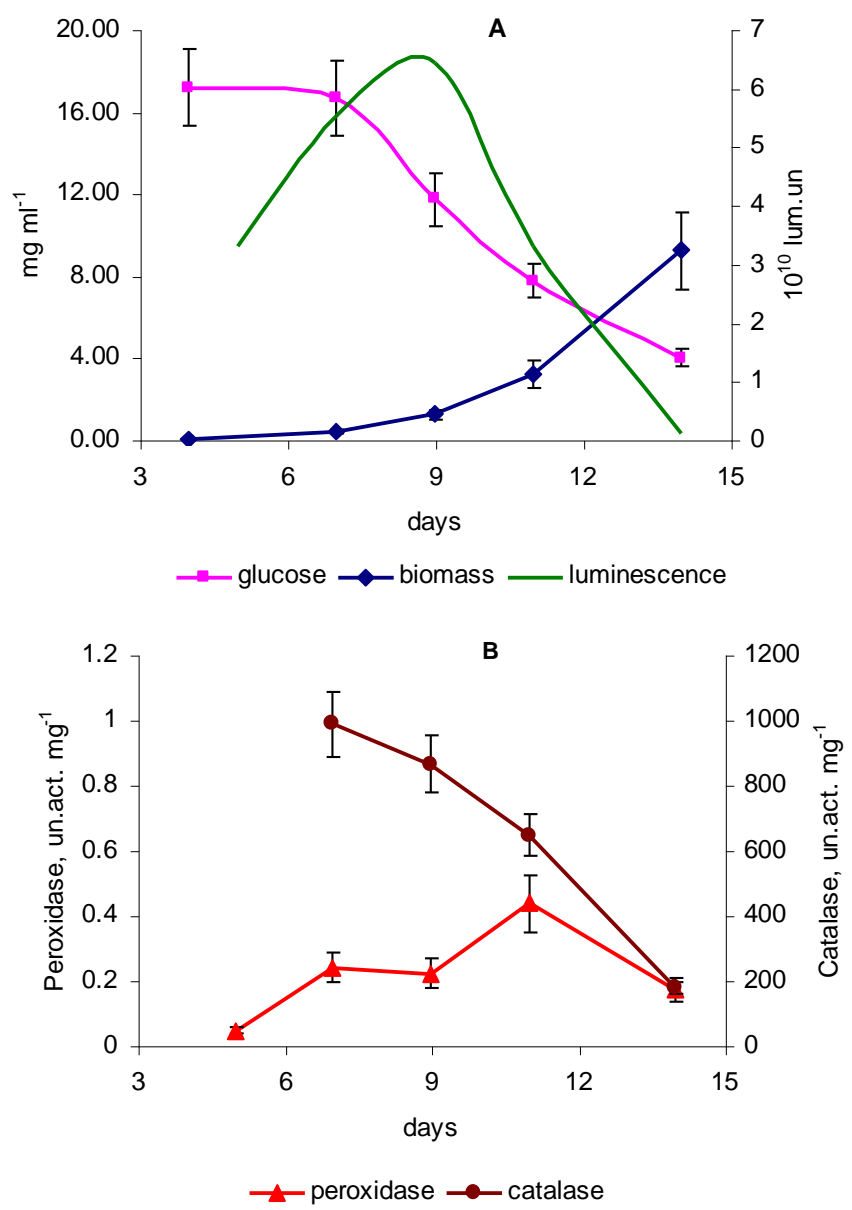

Fig. 1 - A. Time-course data of biomass production, glucose consumption, and changes in bioluminescence during submerged cultivation of Armillaria borealis IBSO 2328. B. Changes in catalase and total peroxidase activities in mycelial extracts during cultivation of the fungus. Catalase and total peroxidase activity were calculated per $1 \mathrm{mg}$ of protein per $1 \mathrm{~mL}$ of extract. Ranges denote the standard deviations of the three trials. 
At 7-9 days of cultivation, fungal biomass began to accumulate rapidly, consuming carbohydrate substrate at a high rate and emitting light of the highest intensity (Fig. 1A). In that period, we detected the highest level of catalase activity and an increase in total peroxidase activity in mycelial extracts (Fig. 1B). In the subsequent experiments, we used the pellets collected in that culture phase.

In submerged culture under orbital stirring, A. borealis grows in the shape of $2-8 \mathrm{~mm}$ oval pellets. The typical appearance of $A$. borealis IBSO 2328 mycelial pellets and their luminescence and a microscopic image of the surface of an individual pellet are shown in Fig. 2. Pellets are of beige color; they get darker over time, and the core takes on an intensely pigmented brown coloration. As a rule, individual pellets differ in the intensity of light emission, which is not related to their size or shape. The periphery of the pellet consists of hyphal bundles, some of them extending for a few millimeters. The inner portion of the pellet is rather dense, consisting of interwoven hyphae and intercellular substance.

As hyphae on the periphery of the pellets were actively growing ones, we focused on them during light and electron microscopic examination. Light microscopy of the pellets showed (Fig. 3) that portions of the hyphae on the periphery of the pellets were intensely pigmented. When stained with fluorescent dye, the pigmented hyphae did not fluoresce and looked dark.

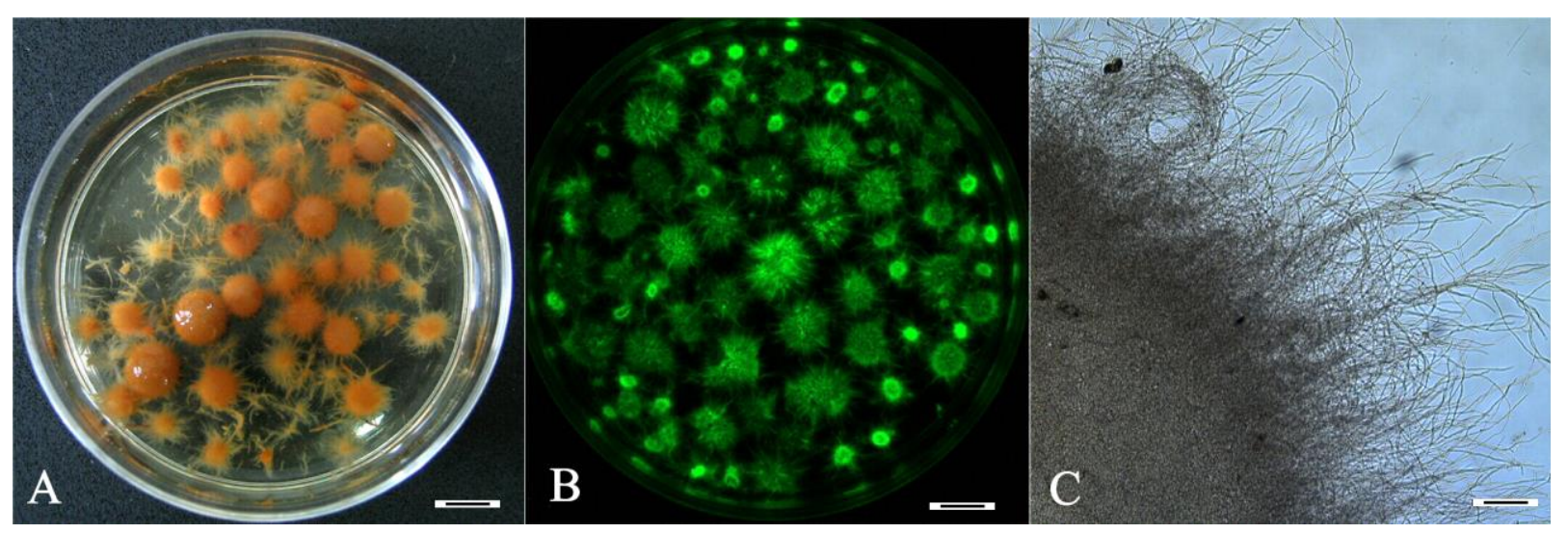

Fig. 2 - A. Reflected-light images of A. borealis IBSO 2328 pellets. B. Images of the pellets taken using their own light in the dark cabinet, in the signal accumulation mode. C. Bright-field image of hyphae on the periphery of the pellet. - Bars $=10 \mathrm{~mm}(\mathrm{~A}, \mathrm{~B})$ and $100 \mu \mathrm{m}(\mathrm{C})$.
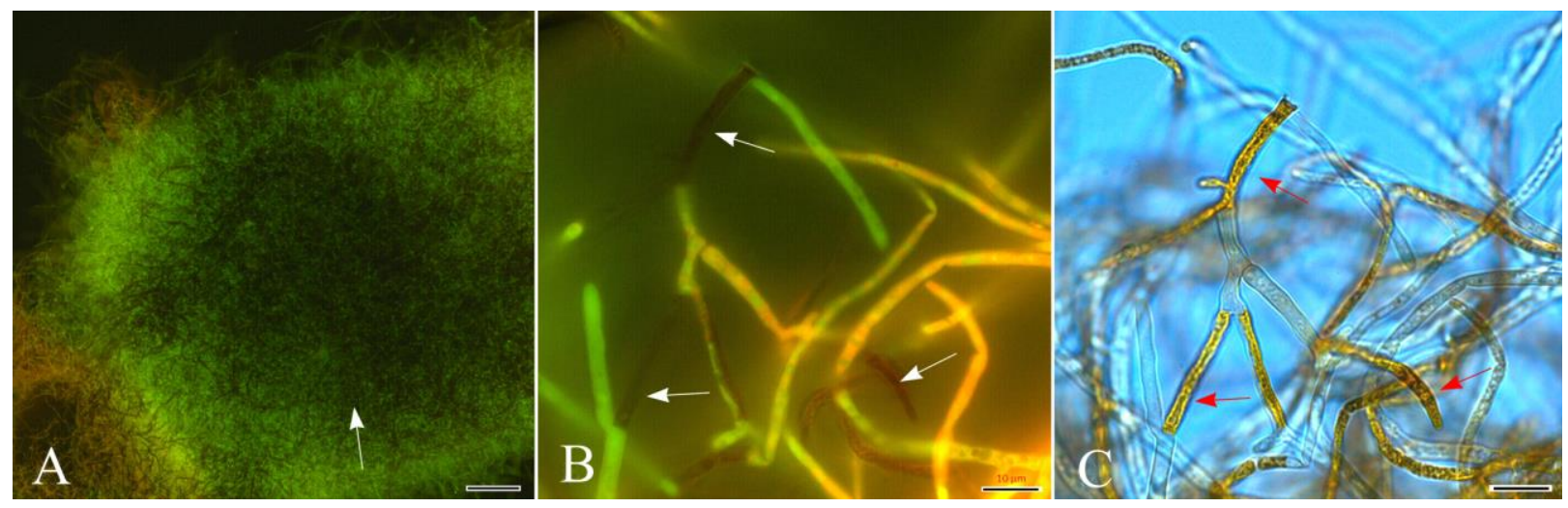

Fig. 3 - Armillaria borealis IBSO 2328 pellets stained with acridine orange dye. A. Flattened pellet under cover glass. B, C. Fluorescing and non-fluorescing hyphae in the fluorescent and bright-field modes of viewing the peripheral region of the pellet. Arrows point to pigmented hyphae impervious to light. - Bars $=100 \mu \mathrm{m}(\mathrm{A})$ and $10 \mu \mathrm{m}(\mathrm{B}, \mathrm{C})$. 
Ultrathin sections of $A$. borealis hyphae (Fig. 4) clearly showed a chitin layer adjacent to the cytoplasmic membrane of the cell wall and a less electron-dense glucan layer, located on the outside of the chitin layer. On the ultrathin sections prepared by the method used in this study, the polysaccharide slime covering the hyphae could not be visualized. On the outer surface of the cell wall, we revealed a pigment layer that had uniform density and structure. On the sections, it looked like differently aligned clusters of rod-shaped structures and sheets, with spaces between them (Fig. 4 A, B). Based on the data reported by different authors, we can state with confidence that this is a melanin layer. In cell cytoplasm, we could see rather large mitochondria, situated under the cytoplasmic membrane, and numerous vacuoles of heterogeneous content.

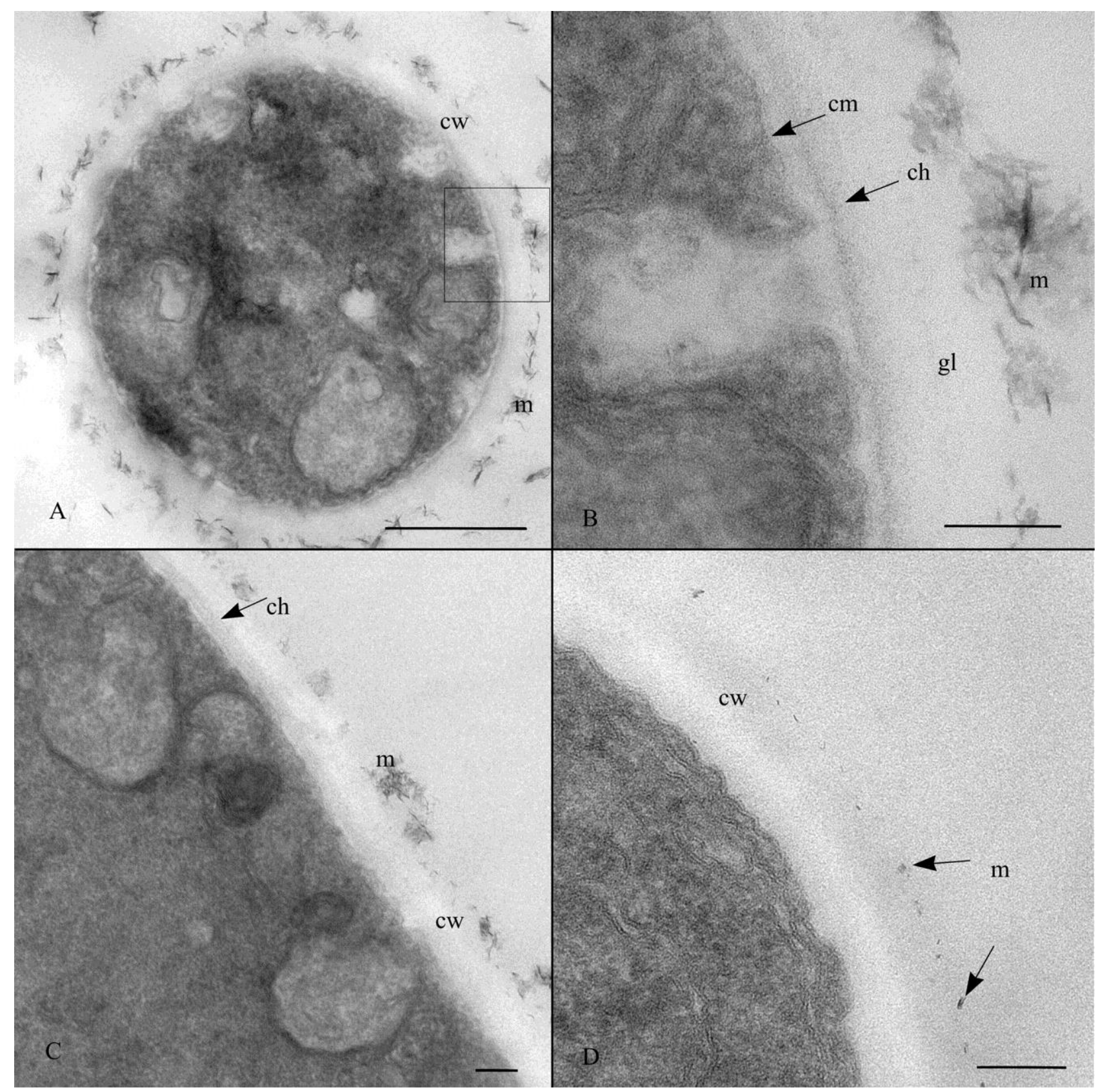

Fig. 4 - TEM images of the Armillaria borealis IBSO 2328 hyphal cross-sections from the peripheral parts of the pellets under different experimental conditions. A. Hyphal section of the pellet incubated in DI water. B. Magnified fragment from image A. C, D. Hyphal sections after $\beta$ glucosidase and chitinase treatments. Abbreviations: ch-chitin layer, cm-cytoplasmic membrane, cw-cell wall, gl- $\beta$-D-glucan layer, m-melanin. - Bars $=500 \mathrm{~nm}(\mathrm{~A})$ and $100 \mathrm{~nm}(\mathrm{~B}-\mathrm{D})$. 


\section{Morphology and luminescence of the mycelium after pellet incubation with $\beta$-glucosidase and chitinase}

Microscopic examination of $A$. borealis IBSO 2328 mycelium incubated with $\beta$-glucosidase or chitinase showed (Fig. 5) that for four hours after the treatment of the pellets with enzymes, most of the peripheral hyphae remained intact. However, there were rather many hyphae with clear indications of osmotic injuries - deformation of cytoplasmic membrane and vacuoles and cytoplasm coagulation.

Electron microscopic examination of pellets incubated with $\beta$-glucosidase revealed perceptible changes in their ultrastructure in the melanin layer outside the cell wall (Fig. 4C). The melanin layer was still present, but distances between pigment clusters had increased. Changes in the ultrastructure of the hyphal cell wall were more substantial in the pellets incubated with chitinase (Fig. 4D). The outer layer of the pigment disappeared or there were scarcely noticeable fragments of that layer; no chitin layer, localized closer to the cytoplasmic membrane, was visible either. Characteristic changes in intracellular ultrastructure were invaginations of cytoplasmic membrane, reduction in vacuole turgor, exhibited as a change in the shape of the vacuoles, and the presence of large deformed multivesicular bodies in the cytoplasm.

Results of studying the level of light emission by A. borealis IBSO 2328 mycelium untreated and treated with the enzymes are shown in Fig. 6A. Mycelial pellets incubated in DI water with $\beta$-glucosidase and chitinase showed increased luminescence intensity compared to the luminescence level of the control pellets, incubated in DI water without these enzymes, for four hours after incubation. These results suggested that by affecting the cell envelope of $A$. borealis hyphae, the enzymes tested in this study stimulated light emission and that chitinase treatment produced a stronger effect.

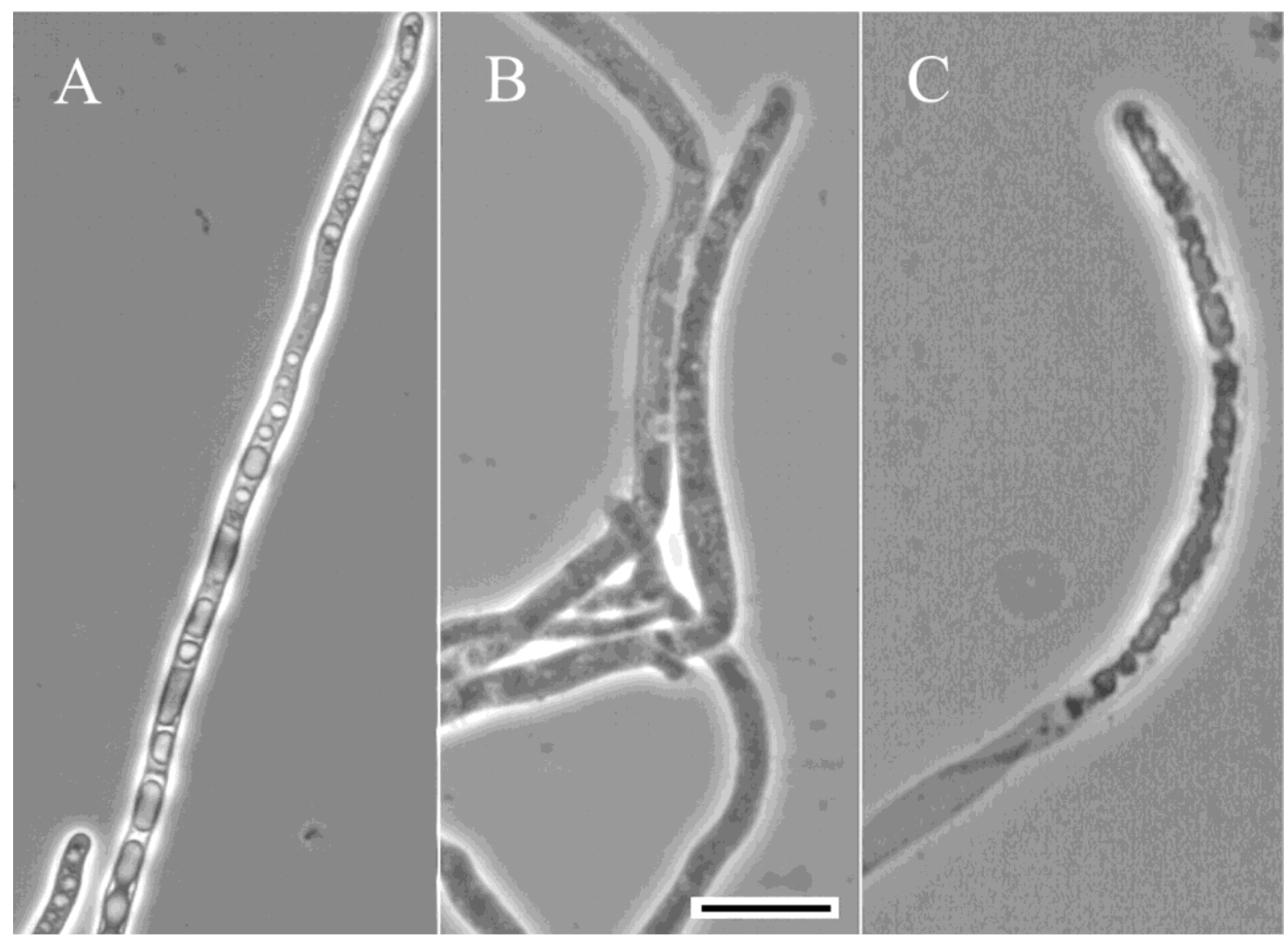

Fig. 5 - Phase-contrast microscopy of the hyphae from the periphery of Armillaria borealis IBSO 2328 pellets. A. Hyphae in DI water. B. Hyphae treated with $\beta$-glucosidase. C. A coagulated hypha after chitinase treatment. - Bar $=10 \mu \mathrm{m}$. 
Detection of the extracellular peroxidase activity of mycelium pellets in vivo and the total peroxidase activity in incubation medium of pellets

Testing of peroxidase activity in vivo showed (Fig. 6B) that after incubation with $\beta$ glucosidase and chitinase, A. borealis IBSO 2328 mycelial pellets produced $20 \%$ and 50\%, respectively, more chromogen than control pellets, which had been incubated in water without these enzymes. However, far more significant differences were revealed in levels of peroxidase activity in pellet incubation media containing the enzymes (Fig. 6B). A very small quantity of the chromogen produced by the oxidative azo-coupling reaction was formed in the control pellet incubation medium. That could result from the insignificant amounts of the enzymes with peroxidase activity in the medium. On the other hand, after incubation of pellets in the medium supplemented with $\beta$-glucosidase and chitinase, chromogen production in these media increased more substantially (12- and 50-fold, respectively).
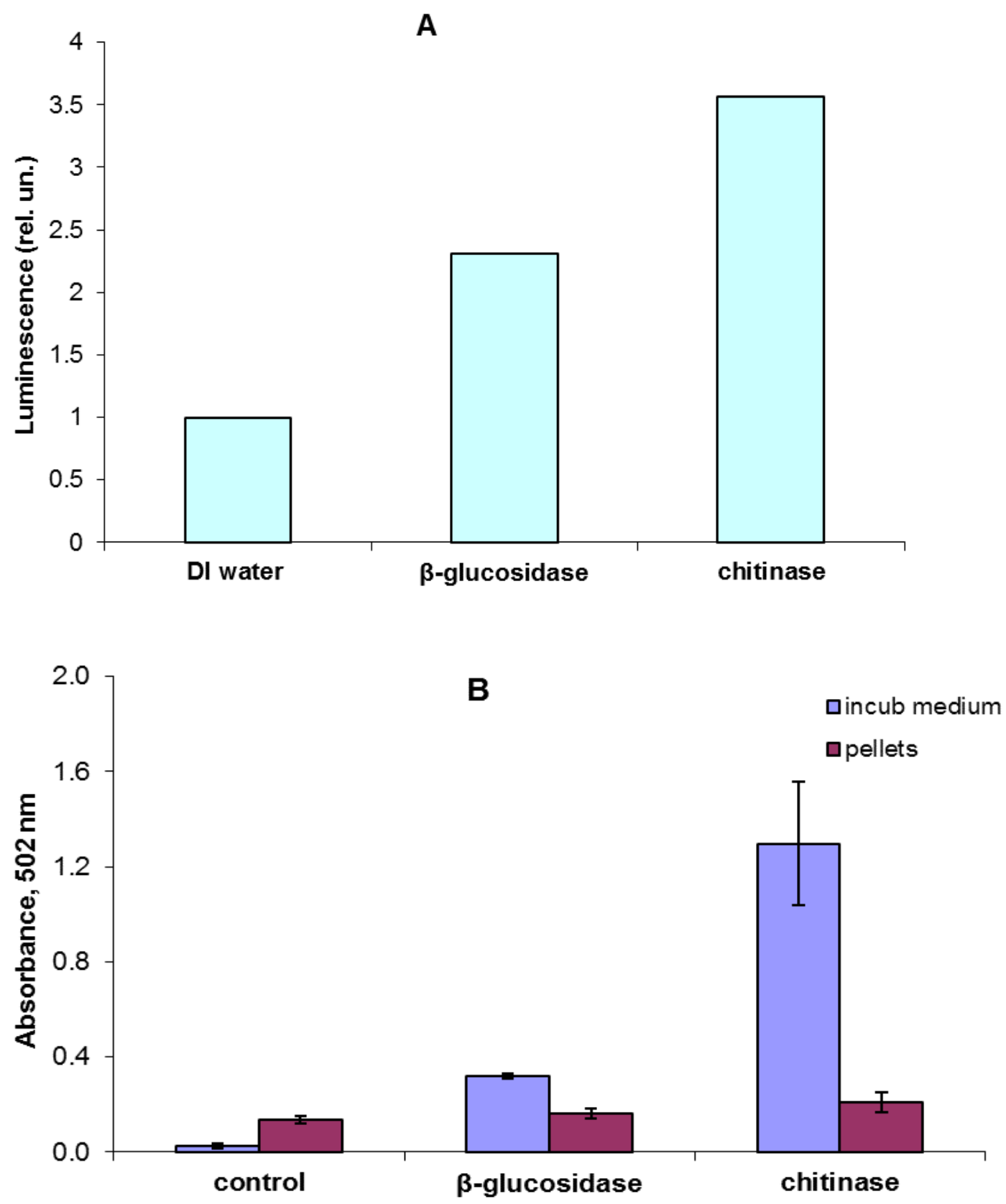

Fig. 6 - Incubations of Armillaria borealis IBSO 2328 pellets with $\beta$-glucosidase and chitinase compared to control samples (incubated in DI water under the same conditions). A. Changes in light emission. B. Peroxidase activity in pellets and incubation medium. The level of luminescence of the treatment samples normalized to the level of luminescence of the pellets in DI water. 


\section{Discussion}

The study of luminous mycelium of A. borealis IBSO 2328 produced the following results. Light and electron microscopy showed that after short-term (4-hour) incubation of pellets in hypoosmotic medium (DI water), the hyphae of $A$. borealis mycelium remained morphologically intact. The structural integrity could be preserved due to resilience of the hyphal cell wall and, hence, due to osmotic pressure remaining balanced. At the same time, incubation of pellets with $\beta$-glucosidase and chitinase caused considerable morphological changes in the mycelium. Hyphae on the periphery of the pellets were exposed to osmotic shock, as indicated by the occurrence of deformed cells with coagulated cytoplasm (Fig. 5). The most noticeable changes were revealed in the structure of the hyphal cell wall. Analysis of results obtained in this study suggested that treatment of mycelium with chitinase produced a more destructive effect than $\beta$-glucosidase treatment. After mycelium was incubated in the medium with $\beta$-glucosidase, the outer pigment layer of the hyphal cell wall was only partially destroyed. After incubation of the pellets in the medium with chitinase, however, neither the chitin layer of the cell wall nor the outer melanin layer were detected in peripheral hyphae of the mycelium. It is well-known that melanin granules are bound to polysaccharides of the cell wall and anchored within the chitin layer (Bull 1970, Eisenman et al. 2005, Nosanchuk et al. 2015). The effect of chitinase on mycelium must have caused not only destruction of the chitin layer of the cell wall but also release of melanin molecules anchored onto chitin fibrils, which were subsequently washed off the surface of hyphae. Results obtained in the present study suggest that as the ultrastructure of the cell walls of peripheral hyphae was disrupted by $\beta$-glucosidase and chitinase, changes were observed in the levels of extracellular peroxidase activity and intensity of light emission of the pellets. The differences revealed here can be explained by the mechanism of the destructive impact of $\beta$-glucosidase and chitinase on the cell envelope of the fungal hyphae. Extracellular peroxidases in fungi can be associated with the cytoplasmic membrane or localized inside or outside the cell wall (Daniel at al. 1989, Ruel \& Joseleau 1991). On the one hand, after the cell envelope integrity is disrupted, peroxidases localized, e.g., on the cytoplasmic membrane must become more readily accessible to exogenous substrates (phenol, 4-AAP). Thus, production of chromogen in azo-coupling reaction must be more effective. At the same time, as the cell envelope of the hyphae is destroyed, fungal extracellular peroxidases are released into the incubation medium. The increase in the chromogen production by the pellets treated with the enzymes should be related to peroxidases immobilized on the cytoplasmic membrane, as they have become more readily accessible to the substrates. After disruption of the hyphal envelope, peroxidases associated with the polysaccharide network and localized closer to the outer boundary of the cell wall diffuse into the external medium. In both cases, extracellular peroxidase activity changes in the same direction. Our experiments showed that levels of light emission by pellets treated with $\beta$-glucosidase and chitinase were 2.5-3.5 times higher than the level of luminescence of the control pellets. We propose two possible mechanisms of this effect. On the one hand, the increase in the luminescence intensity of the enzyme-treated pellets may be attributed to destruction of the surface pigment (melanin) layer of the hyphae, which can serve as an optical filter. Melanin pigments are known to absorb light in a wide range of wavelengths (Meredith \& Sarna, 2006). On the other hand, as the enzyme-treated fungus loses a considerable portion of extracellular peroxidases, the effectiveness of its antioxidant defense system will be reduced. Then, the increase in the level of luminescence of mycelial pellets may be a compensatory response of the fungus, aimed at neutralizing active oxygen radicals that are formed under stress conditions by light emission. In a previous study, we put forward the hypothesis suggesting that luminescence of higher fungi might be an additional mechanism of their antioxidant defense against the damaging effects of ROS (Mogilnaya et al. 2015, 2016).

\section{Disclosure}

The authors declare no conflicts of interest. All the experiments undertaken in this study comply with the current laws of the country where they were performed. 


\section{Acknowledgments}

This work was supported by the state budget allocated to the fundamental research at the Russian Academy of Sciences (project no. 0356-2016-0709) and Program No.II.2 «Integration and Development» of the Siberian Branch of the Russian Academy of Sciences (project no. 0356-20150103).

\section{References}

Bowman SM, Free SJ. 2006 - The structure and synthesis of the fungal cell wall. BioEssays 28, 799-808.

Breitenbach M, Weber M, Rinnerthaler M, Karl T, Breitenbach-Koller L. 2015 - Oxidative stress in fungi: its function in signal transduction, interaction with plant hosts, and lignocellulose degradation. Biomolecules 5, 318-342.

Bull AT. 1970 - Chemical composition of wild-type and mutant Aspergillus nidulans cell walls. The nature of polysaccharide and melanin constituents. Journal of General Microbiology 63, 75-94.

Carmona-Ribeiro AM, Prieto T, Nantes IL. 2015 - Nanostructures for peroxidases. Frontiers in Molecular Biosciences 2, 50 doi: 10.3389/fmolb.2015.00050.

Conesa A, Punt PJ, van den Hondel CA. 2002 - Fungal peroxidases: molecular aspects and applications. Journal of Biotechnology 93, 143-158.

Daniel G, Nilsson T, Pettersson B. 1989 - Intra- and extracellular localization of lignin peroxidase during the degradation of solid wood and wood fragments by Phanerochaete chrysosporium by using transmission electron microscopy and immuno-gold labeling. Applied and Environmental Microbiology 55, 871-881.

De Castro C, Del Valle P, Rua J, Garsia-Armesto MR et al. 2013 - Antioxidant defence system during exponential and stationary growth phases of Phycomyces blakesleeanus: Response to oxidative stress by hydrogen peroxide. Fungal Biology 117, 275-287.

Ene IV, Walker LA, Schiavone M, Lee KK et al. 2015 - Cell wall remodeling enzymes modulate fungal cell wall elasticity and osmotic stress resistance. mBio 6(4):e00986-15. doi:10.1128/mBio.00986-15.

Eisenman HC, Nosanchuk JD, Webber JBW, Emerson RJ, Camesano TA, Casadevall A. 2005 Microstructure of cell wall-associated melanin in the human pathogenic fungus Cryptococcus neoformans. Biochemistry 44, 3683-3693.

Fesel PH, Zuccaro A. 2016 - $\beta$-gluican: Crucial component of the fungal cell wall and elusive MAMP in plants. Fungal Genetics and Biology 90, 53-60.

Feofilova EP. 2010 - The fungal cell wall: modern concepts of its composition and biological function. Microbiology (Moscow) 79, 711-720.

Free SJ. 2013 - Fungal cell wall organization and biosynthesis. Advances in Genetics 81, 33-82.

Gessler NN, Aver'yanov AA, Belozerskaya TA. 2007 - Reactive oxygen species in regulation of fungal development. Biochemistry (Moscow) 72, 1091-1109.

Gessler NN, Egorova AS, Belozerskaya TA. 2014 - Melanin pigments of fungi under extreme environmental conditions (review). Applied Biochemistry and Microbiology 50, 105-113.

Hastings JW, Weber G. 1963 - Total quantum flux of isotopic sources. Journal of the Optical Society of America 53, 1410-1415.

Hegnauer H, Nyhle NL, Rast D. 1985 - Ultrastructure of native and synthetic Agaricus bisporus melanins. Implications as to compartmentation of melanogenesis in fungi. Experimental Mycology 9, 1-29.

Hofrichter M, Ullrich R, Pecyna MJ, Liers C, Lunde T. 2010 - New and classic families of secreted fungal heme peroxidases. Applied Microbiology and Biotechnology 87, 871-897.

Knop D, Yarden O, Hadar Y. 2015 - The ligninolytic peroxidases in the genus Pleurotus: divergence in activities, expression, and potential applications Applied Microbiology and Biotechnology 99, 1025-1038. 
Koroleva OV, Kulikova NA, Alekseeva TN, Stepanova EV et al. 2007 - A comparative characterization of fungal melanin and the humin-like substances synthesized by Cerrena maxima 0275. Applied Biochemistry and Microbiology 43, 61-67.

Latge' J-P, Beauvais A. 2014 - Functional duality of the cell wall. Current Opinion in Microbiology 20, 111-117.

Medvedeva SE, Artemenko KS, Krivosheenko AA, Rusinova AG et al. 2014 - Growth and light emission of luminous basidiomycetes cultivated on solid media and in submerged culture. Mycosphere 5, 565-577.

Meredith P, Sarna T. 2006 - The physical and chemical properties of eumelanin. Pigment Cell Research 19, 549-659.

Mihail JD, Bruhn JN. 2007 - Dynamics of bioluminescence by Armillaria gallica, A. mellea and A. tabescens. Mycologia 99, 341-350.

Mihail JD. 2013 - Comparative bioluminescence dynamics among multiple Armillaria gallica, A. mellea, and A. tabescens genets. Fungal Biology 117, 202-210.

Mihail JD. 2015 - Bioluminescence patterns among North American Armillaria species. Fungal Biology 119, 528-537.

Mogilnaya OA, Ronzhin NO, Medvedeva SE, Bondar VS. 2015 - Total peroxidase and catalase activity of luminous basidiomycetes Armillaria borealis and Neonothopanus nambi in comparison with the level of light emission. Applied Biochemistry and Microbiology 51, 419-424.

Mogilnaya OA, Ronzhin NO, Bondar VS. 2016 - Comparative evaluation of total peroxidase and catalase activities during light emission of luminous fungus Neonothopanus nambi. Mycosphere 7, 499-510.

Nosanchuk JD, Stark RE, Casadevall A. 2015 - Fungal melanin: what do we know about structure? Frontiers in Microbiology 6, 1463. doi: 10.3389/fmicb.2015.01463

Osińska-Jaroszuk M, Jarosz-Wilkołazka A, Jaroszuk-Ściseł J, Szałapata K et al. 2015 Extracellular polysaccharides from Ascomycota and Basidiomycota: production conditions, biochemical characteristics, and biological properties. World Journal of Microbiology and Biotechnology 31, 1823-1844.

Pollegioni L, Tonin F, Rosini E. 2015 - Lignin-degrading enzymes. FEBS Journal 282, 1190-1213.

Ruel K, Joseleau J-P. 1991 - Involvement of an extracellular glucan sheath during degradation of populus wood by Phanerochaete chrysosporium. Applied and Environmental Microbiology 57, 374-384.

Ruiz-Duenas FJ, Martınez AT. 2010 - Structural and functional features of peroxidases with a potential as industrial biocatalysts. In: Torres E, Ayala M (eds), Biocatalysis based on heme peroxidases. Springer-Verlag, Berlin.

Sáez-Jiménez V, Acebes S, Guallar V, Martínez A, Ruiz-Dueñas F. 2015 - Improving the oxidative stability of a high redox potential fungal peroxidase by rational design. PLoS ONE 10, e0124750. doi: 10.1371/journal.pone.0124750.

Shimomura O. 2006 - Bioluminescence: chemical principles and methods. World Scientific Publishing, Singapore.

Tomanek L. 2015 - Proteomic responses to environmentally induced oxidative stress. The Journal of Experimental Biology 218, 1867-1879.

Upadhyay S, Xu X, Lowry D, Jackson JC, Roberson RW, Lin X. 2016 - Subcellular compartmentalization and trafficking of the biosynthetic machinery for fungal melanin. Cell Reports 14, 2511-2518.

Wong D. 2009 - Structure and action mechanism of ligninolytic enzymes. Applied Biochemistry and Biotechnology 157, 174-209.

Zhong J, Frases S, Wang H, Casadevall A, Stark RE. 2008 - Following fungal melanin biosynthesis with solid-state NMR: biopolymer molecular structures and possible connections to cell-wall polysaccharides. Biochemistry 47, 4701-4710. 
Zink P, Zink D. 1989 - Studies on the colouring matter of blue-stain fungi Part 2. Electron microscopic observations of the hyphae walls. Holzforschung 43, 371-374. 